\title{
Lactancia materna: rompiendo las barreras
}

\author{
Paulina Velasco Riestra, Adelaida Caicedo Fajardo, José Antonio Navarro \\ Rodríguez, María Fernanda Tejada Pineda y Mónica Jackeline Armas Neira
}

\begin{abstract}
Resumen
La lactancia materna es la intervención más costo-efectiva para la salud de los recién nacidos y de sus madres. Es una práctica fisiológica que reduce el riesgo de enfermedades e infecciones del recién nacido, además, mejora su desarrollo y su crecimiento. Simultáneamente, disminuye el riesgo materno de cáncer de mama, de sangrado posterior al parto, confiere protección anticonceptiva y mejora el vínculo emocional entre la madre y el hijo. A pesar de estos beneficios, estadísticas del país demuestran una deficiencia en esta práctica. En este artículo se ahondan los beneficios de la lactancia, sus mitos y realidades provenientes de creencias sociales, con el fin de disminuir las barreras que limitan la lactancia materna y así, mejorar la salud de madres e hijos.
\end{abstract}

Palabras clave: lactancia, leche materna, recién nacido, salud materna.

\section{BREASTFEEDING: BREAKING DOWN BARRIERS}

\begin{abstract}
Breastfeeding is the most cost-effective intervention regarding the health of newborns and their mothers. It is a physiological practice that reduces the risk of diseases and infections of the newborn, and improves its development and growth. It simultaneously reduces the maternal risk of breast cancer, bleeding after childbirth, and it provides contraceptive protection and improves the emotional bond between mother and child. Despite these benefits, Mexican statistics show a deficiency in this practice. This article delves into the benefits of breastfeeding, myths and realities that have emerged from social beliefs around it, in order to reduce the barriers that limit breastfeeding and thus improve the health of mothers and children.
\end{abstract}

Keywords: breastfeeding, human milk, newborn, maternal health.

Recepción: 14/12/2020. Aprobación: 12/03/2021.

http://doi.org/10.22201/cuaieed.16076079e.2021.22.4.8 
"Lactancia materna: rompiendo las barreras" Paulina Velasco Riestra, Adelaida Caicedo Fajardo, José Antonio Navarro Rodríguez, María Fernanda Tejada Pineda y Mónica Jackeline Armas Neira Vol. 22, Núm. 4, julio-agosto 2021 Revista Digital Universitaria

\section{Paulina Velasco Riestra}

paulina.velasco.riestra@gmail.com orcid.org/0000-0001-7145-2619

Actualmente se encuentra en formación como médico cirujano por el Tecnológico de Monterrey, y está cursando el internado en el Centro Médico ABC, el Hospital Materno Pediátrico de Xochimilco y el Hospital General de Xoco. Cuenta con experiencia en investigación de ciencias básicas en el departamento de medicina pulmonar del MD Anderson en Houston, Texas. Ha paticipado de manera activa en mesas directivas de grupos estudiantiles con fines altruistas para apoyo a la comunidad y sociedades académicas y estudiantiles con temática médica. Asimismo, cuenta con certificación de primeros auxilios psicológicos. Tiene un interés activo en la pediatría.

\section{Adelaida Caicedo Fajardo}

adecaicedo@hotmail.com orcid.org/0000-0002-8109-0903

Estudiante de Medicina en el Instituto Tecnológico y de Estudios Superiores de Monterrey, con amplio interés en la salud pública y salud global. Actualmente es Médico Interno de Pregrado en el Centro Médico ABC, Hospital Materno Pediátrico de Xochimilco y Hospital General Xoco. Practicante en el proyecto de investigación novus, sobre mentoreo en el área de ciencias básicas, con un enfoque en bioquímica, durante un año. Tiene experiencia trabajando en equipos, coordinando como parte de la mesa directiva de varios grupos estudiantiles, organizando eventos y actividades con diferentes propósitos en torno a la salud y el bienestar de la comunidad. Cuenta con voluntariados en torno a la salud, la sociedad y el medio ambiente, así como con un diplomado de liderazgo por el Tecnológico de Monterrey.

José Antonio Navarro Rodríguez

a01335659@gmail.com

orcid.org/0000-0003-3830-2920

Médico Cirujano en formación por el Instituto Tecnológico y de Estudios Superiores de Monterrey, campus Ciudad de México, actualmente está laborando como Médico Interno de Pregrado en el Centro Médico ABC. Tiene antecedentes laborales como Coordinador de Mentorías en el Campus del al proyecto novus en donde se desempeñó como revisor del desarrollo del proyecto y evaluador de las actividades de las materias de Biología Celular y Bioquímica. Tiene experiencia como Coordinador del Programa de Asesorías entre alumnos por parte de la Sociedad de Alumnos de la Escuela de Medicina, y fue Fundador y Presidente del Grupo Estudiantil de Medicina Interna y Pensamiento Crítico de su alma mater, organizando espacios de difusión y práctica, apoyándose en ponencias y simuladores clínicos. Está interesado en la docencia, la atención a las personas que viven con una discapacidad, la salud mental y las neurociencias.

\section{María Fernanda Tejada Pineda}

A01337088@itesm.mx orcid.org/0000-0002-4890-803X

Estudiante de medicina en el Instituto Tecnológico de Estudios Superiores de Monterrey; actualmente cursa el internado de pregrado. Su pasión por la neurocirugía la llevó a fundar en el año 2018 el capítulo de estudiantes de medicina de la American Association of Neurological Surgeons (AANs) en el campus Ciudad de México. Organizó como coordinadora principal el Primer Simposio Nacional de Neuroanatomía, el cual fue avalado por la Sociedad Mexicana de Cirugía Neurológica, el Instituto Nacional de Cancerología y el Hospital Ángeles de Acoxpa. Participó como expositora con el tema "Inmunoterapia para el tratamiento de glioblastomas" en el xxv Congreso Mexicano e Internacional de Cirugía Neurológica 2019 y en el Journal Club organizado por el capítulo AANS UNAM, con el tema "El quirófano del futuro versus el futuro del quirófano". Publicó el artículo "Treatment and outcome in 12 cases of olfactory neuroblastoma at Mexico's National Cancer Institute: A retrospective clinical analysis and literature review". Colaboró con el capítulo de "Microsurgical Anatomy of the White Matter Tracts of Brainstem and Cerebellum" en la nueva edición del libro Principles of Neuro-Oncology: Brain and Skull Base de Springer. 
"Lactancia materna: rompiendo las barreras"

Paulina Velasco Riestra, Adelaida Caicedo Fajardo, José Antonio Navarro Rodríguez,

María Fernanda Tejada Pineda y Mónica Jackeline Armas Neira

Vol. 22, Núm. 4, julio-agosto 2021

Revista Digital Universitaria

Mónica Jackeline Armas Neira

A01019195@itesm.mx

orcid.org/0000-0003-1750-2851

Estudiante de medicina en el Tecnológico de Monterrey con un Bachelor en ciencias previo en Biología, por parte de Baylor University. Cuenta con experiencia en investigación de ciencias básicas en el Departamento de Pulmonar del MD Anderson en Houston, Texas. Tiene una publicación sobre el uso de medicina alternativa y complementaria en pacientes pediátricos durante un programa de mentoreo en Baylor Scott \& White Health. Actualmente cursa un año de internado con sede en el Centro médico ABC y Hospital Materno pediátrico de Xochimilco.

\section{Introducción}

Los seres humanos necesitamos de nuestra madre desde el momento de nuestro nacimiento para alimentarnos, continuamos con esta acción por meses hasta tener la capacidad de nutrirnos con otros alimentos y así, poder continuar con nuestro proceso de crecimiento y desarrollo. Sin embargo, la lactancia materna es más que sólo alimentación, es un proceso no instintivo, construido socialmente, limitado o expandido por factores culturales, religiosos, de aprendizaje, o por fuentes de información moderna, etc. Esto se ejemplifica desde las antiguas civilizaciones con Hera en Grecia, Isis en Egipto y Rumia en Roma, deidades conocidas por sus atributos de fertilidad y lactancia (Rodríguez García, 2015). Sin embargo, esta no deja de ser un acto que involucra el bienestar de la madre y el goce de su cuerpo para compartirlo con el recién nacido; también, la lactancia toma en cuenta no sólo la salud y las necesidades del bebé, sino las de ambos.

Respecto a lo anterior, ¿Las creencias alrededor de la lactancia son ciertas? Afortunadamente, en este trabajo se busca dar respuesta a esta interrogante mediante la investigación científica y la recopilación de los beneficios de la lactancia materna y su impacto en el recién nacido, en la madre, e incluso, en la sociedad. 


\section{Lactancia}

Victora et al. mencionan que, si se cumpliera con la lactancia materna de forma exclusiva en todos los bebés del mundo, se podrían salvar alrededor de 823000 vidas anualmente (2016). Pero ¿Cómo lograr esta ambiciosa meta?, en primer lugar, se puede empezar por conocer las recomendaciones propuestas de organismos como la Organización Mundial de la Salud (oms) y el Fondo de las Naciones Unidas para la Infancia (UNICEF) para aprovechar la lactancia de la mejor manera posible (Organización Mundial de la Salud [OMs], 2020), por ejemplo:

- Iniciar la lactancia en la primera hora de vida.

- No complementar la lactancia con agua.

- Dejar que el bebé decida la frecuencia con la que quiere recibirla.

- No utilizar biberones, chupones ni otros aditamentos para alimentarlo.

- Dar lactancia materna exclusiva durante al menos los primeros seis meses de vida para, posteriormente, integrar alimentos complementarios, pero manteniéndola hasta los dos años, o más.

\section{Epidemiología}

Se dice que la lactancia materna exclusiva es la intervención más costo-efectiva para la sobrevida y para la salud de los recién nacidos. Algunos de los beneficios de la lactancia para los bebés son: un crecimiento óptimo, un adecuado desarrollo cerebral, disminución del riesgo de enfermedades, beneficios psicológicos y emocionales, entre muchísimas más ventajas. Incluso, hay beneficios para la madre, como un menor riesgo de presentar hemorragias después del parto, la disminución del riesgo de desarrollar cáncer de mama y de ovario, entre otros beneficios que mencionaremos más adelante.

Sin embargo, a pesar de las constantes recomendaciones, como país nos hemos quedado atrás. Según la Encuesta Nacional de Niños, Niñas y Mujeres, en México, sólo uno de cada tres bebés se alimenta con lactancia materna exclusiva hasta los seis meses de edad (Instituto Nacional de Salud Pública et al., 2016). Este es un dato alarmante ya que no se están alcanzando los beneficios que la lactancia materna ofrece a corto y a largo plazo.

Desafortunadamente, existen barreras que dificultan o impiden a las madres ofrecer la lactancia, pero ¿Cuáles predominan en nuestra población? En el 2013 se realizó una investigación para identificarlas, entre ellas se encuentran que las madres piensan que su leche no contiene los suficientes nutrientes para su bebé, también, otros factores importantes son las restricciones laborales, las recomendaciones inadecuadas por parte de los proveedores de salud, la estigmatización social, e incluso las políticas públicas que dificultan la lactancia (Bonvecchio et al., 2016). 
Asimismo, el tabú que existe alrededor de una madre amamantando en público obstaculiza el acto; para combatir esto, la educación y la desestigmatización alrededor de la lactancia materna son medidas básicas y necesarias para modificar estas limitantes, y asimismo, para reconocer el derecho a la lactancia como un derecho humano (Comisión Nacional de los Derechos Humanos [CNDH], 2018). Para más información sobre mitos y realidades, se puede consultar la imagen 1.

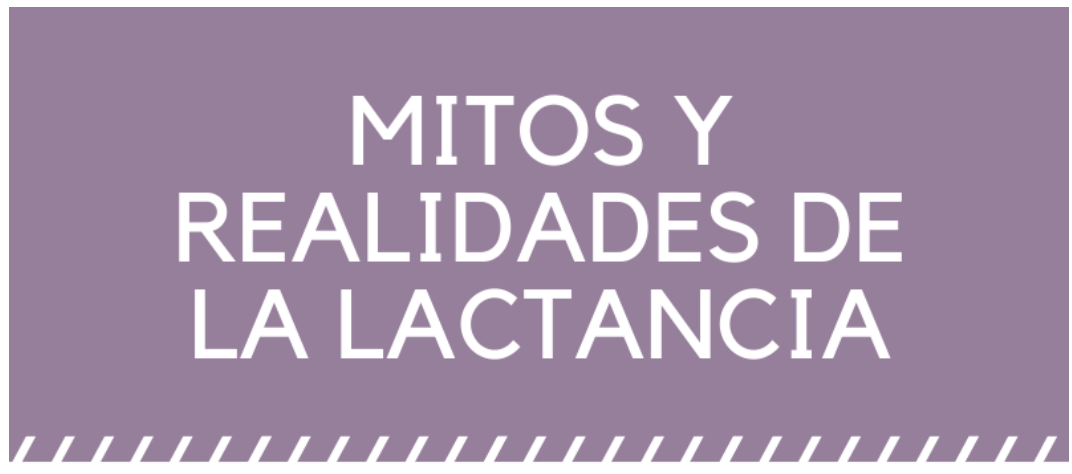

Imagen 1. Mitos y realidades. Autoría de María Fernanda Tejada Pineda.

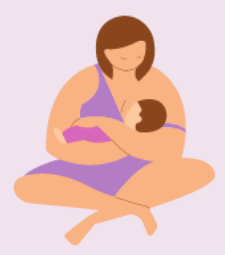

"No tengo suficiente leche"

La principal causa descrita para el abandono de la lactancia es la creencia de no tener suficiente producción, sin embargo, en la mayoria de los casos las mamás tienen producción adecuada

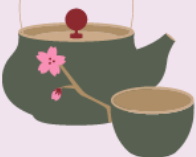

"Si mi bebé tiene cólicos, le debo dar té para aliviarlos"

La OMS recomienda la lactancia materna exclusiva hasta los 6 meses, no se debe complementar con agua ni té.

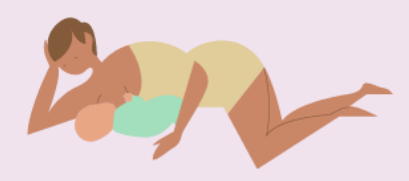

\section{"La leche materna deja de servir} después de los 6 meses"

La leche materna se adapta a las necesidades del niño, mientras exista un estimulo para la producción de leche, se va a seguir produciendo. La OMS recomienda continuar con la lactancia hasta los dos o más años de edad.

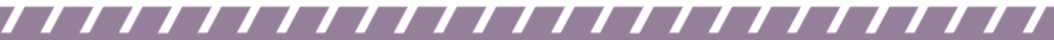




\section{La leche materna}

Hasta el siglo xIx se tenía poco conocimiento sobre la composición de la leche humana, incluso se alimentaba a los bebés con leche de distintas especies, lo cual ocasionó una mayor mortalidad infantil (Talayero, 2004). ¿Qué hace a la leche humana tan especial? La respuesta es su multifuncionalidad ya que la leche materna se considera bioactiva, es decir, las sustancias que la componen cumplen funciones diferentes a nivel celular. Además, la leche cambia su composición a lo largo del tiempo, y la podemos clasificar en 3 subtipos: calostro, leche de transición y leche madura.

Inicialmente, se produce el líquido denominado calostro que contiene poca lactosa, factores de crecimiento, sodio, agua y minerales. Tiene una coloración amarillenta y aparenta ser escasa, aunque cubre las necesidades esenciales de un recién nacido. Es importante mencionar que entre más se estimulan las mamas con la succión del bebé, más aumenta la producción. Después, tenemos la leche de transición, ésta es el líquido transitorio entre el calostro y la leche madura y es la más variada en su contenido: inicia con una coloración amarillenta para después tornarse blanquecina, se produce de manera más abundante, pero sin superar la cantidad de la leche madura y aporta un poco más de calorías que el calostro; además se amolda a las necesidades del bebé hasta llegar a la leche madura. Ésta última se establece después del primer mes, tiene un aspecto blanquecino y da un mayor aporte energético para un bebé que comienza a tener una mayor actividad; contiene los nutrientes necesarios para ser la fuente única de alimentación durante los primeros seis meses de vida; y en este líquido, encontramos grasas, proteínas y azúcares cuyas cantidades varían acorde al tiempo y las necesidades específicas (Witkowska Zimny et al., 2017).

Además de lo previamente descrito, la leche materna también tiene elementos como sales biliares, sustancias necesarias para la digestión de grasas; contiene enzimas, factores de crecimiento, hormonas e incluso bacterias que ayudan a la función digestiva y al desarrollo gastrointestinal del recién nacido. De igual forma, contiene componentes del sistema inmunológico que protegen a los bebés de las infecciones más comunes (Ballard et al., 2013).

\section{Contraindicaciones de la lactancia materna}

Son contadas las razones por las cuales la lactancia se encuentra contraindicada, algunas, descritas en la revisión de Díaz, son (2012):

- La infección por el Virus de la Inmunodeficiencia Humana (VIH), aunque, es importante mencionar que, en países en vías de desarrollo, la balanza favorece a la lactancia. Esto se debe a que es preferible el riesgo de infección que una desnutrición, además, en estos países, por la falta de acceso al agua, pueden existir otros riesgos causados por una mala preparación, utilización y administración de las fórmulas. 
"Lactancia materna: rompiendo las barreras" Paulina Velasco Riestra, Adelaida Caicedo Fajardo, José Antonio Navarro Rodríguez, María Fernanda Tejada Pineda y Mónica Jackeline Armas Neira Vol. 22, Núm. 4, julio-agosto 2021 Revista Digital Universitaria

- Enfermedades metabólicas del niño como la galactosemia, enfermedad genética donde no se puede ingerir un tipo de azúcar llamada galactosa, la cual se encuentra en la leche; ya que puede acumularse en sus órganos y afectarlos. O la deficiencia congénita de lactasa que vuelve al bebé intolerante a la lactosa ya que es incapaz de digerirla debido a la ausencia de este azúcar en su organismo.

- El consumo de fármacos como quimioterapéuticos o radiofármacos con fines terapéuticos o diagnósticos debido a que alteran la calidad de la leche.

- El consumo de drogas como heroína, cocaína, metanfetaminas y marihuana por la madre, ya que pueden tener consecuencias nocivas para el bebé.

Por lo anterior, el satanizar las fórmulas es contraproducente en estos casos, pues resultan útiles cuando no es posible llevar a cabo la lactancia. Pero, es necesario tener en cuenta que las fórmulas nunca van a superar o a igualar las propiedades de la leche materna, además de que representan un gasto ambiental ahorrable en la mayoría de los casos.

\section{Beneficios de la lactancia}

¿Por qué se recomienda tanto la lactancia? Se han descrito beneficios a corto y a largo plazo para la salud del bebé, estos se resumen en las imágenes 2 y 3 . A continuación mencionaremos los beneficios más relevantes para la madre.

A corto plazo, algunos beneficios de la lactancia es que ayuda al cuerpo materno a regresar a su estado basal o a su estado previo del embarazo; por ejemplo, se ha demostrado que el útero regresa más rápido a su tamaño original en mujeres lactantes en comparación con aquellas que no, esto a su vez, disminuye el riesgo de un sangrado posterior al parto y, por consecuencia, el riesgo de anemia (Abedi et al., 2016). Lactar, también, retrasa la ovulación por la presencia de la hormona productora de leche llamada prolactina. Entre más seguido se amamante al bebé, habrá una mayor liberación de prolactina y es así como el cuerpo interpreta que aún no está listo para tener otro bebé. De esta forma, se prolonga el tiempo entre cada embarazo, y se reducen complicaciones futuras (Berens, 2020). Sin embargo, es importante recalcar que la lactancia no es un método anticonceptivo 100\% efectivo, es decir, un embarazo es posible a pesar de estar lactando.

A largo plazo, el riesgo de desarrollar cáncer de mama y de ovario disminuye cuando la mujer amamanta por más de un año (Chowdhury et al., 2015). Asimismo, tiene efectos protectores sobre el corazón y los vasos sanguíneos, pues se ha visto que estas mujeres tienen menor riesgo de tener un infarto en la menopausia (Nguyen et al., 2017). 
Imagen 2. Beneficios de la lactancia en la salud materna. Autoría de María Fernanda Tejada Pineda.

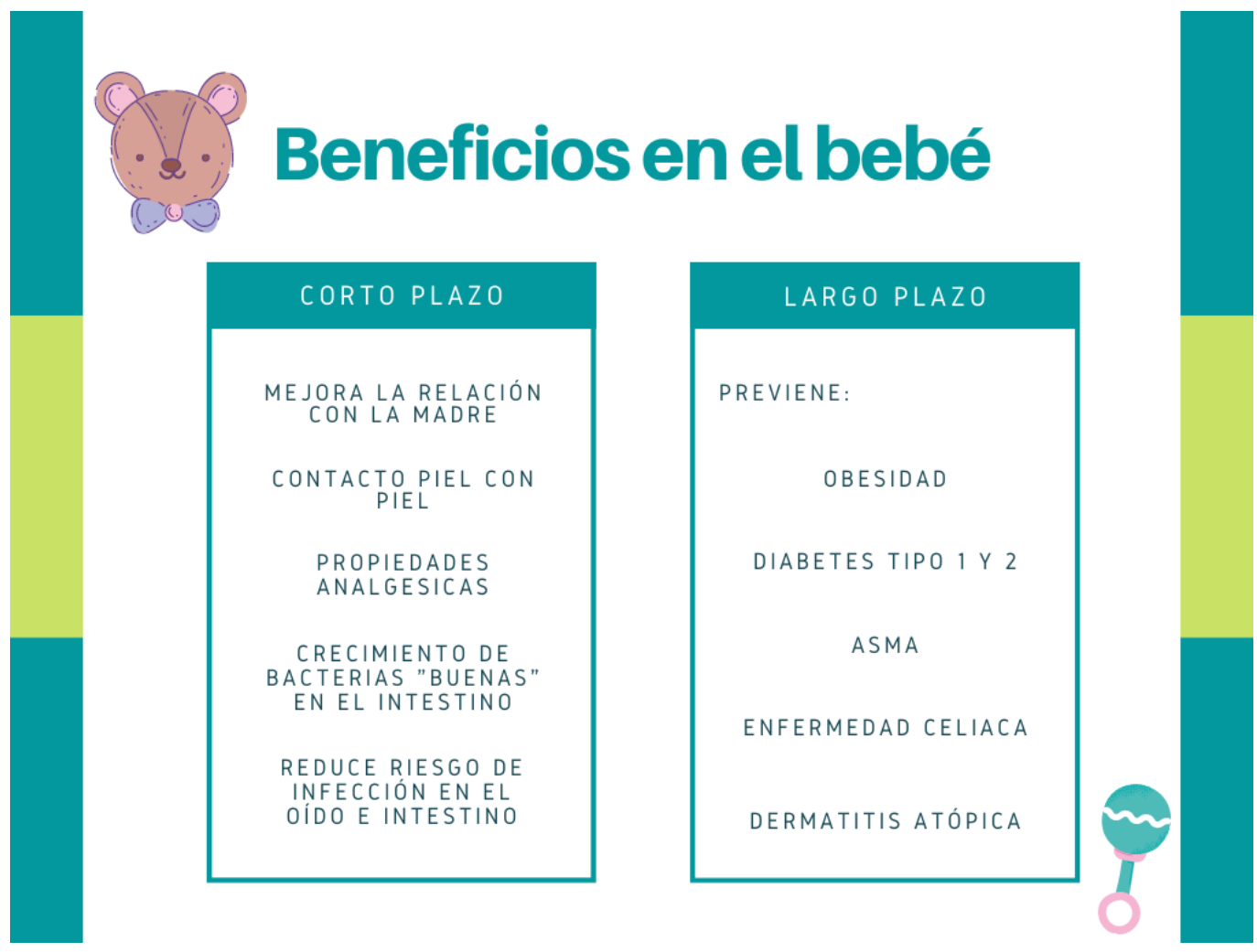

Pasando a los beneficios para el bebé, el contacto piel con piel, que involucra la lactancia, va de la mano con su desarrollo emocional y social. Mediante este contacto se establece una relación cercana madre-hijo (Moore et al., 2016) la cual beneficia la salud mental de ambos y fomenta la producción de más leche. Además, cuenta con propiedades analgésicas, pues los bebés que son amamantados cuentan con menos estrés durante procedimientos dolorosos, en contrario con aquellos que son alimentados con fórmula (Carbajal et al., 2003).

La lactancia promueve el crecimiento de bacterias benéficas que forman parte del tracto digestivo y de la piel. La colonización de estas bacterias, a una edad temprana, promueve el desarrollo del sistema inmunológico, lo que previene tanto enfermedades metabólicas como gastrointestinales y fomenta el desarrollo del sistema inmune y neuronal (Stiemsma, 2018). Adicionalmente, la lactancia tiene un efecto protector sobre las infecciones y las enfermedades provocadas por respuestas inmunológicas exageradas, como lo son el asma y la dermatitis (American Academy of Pediatrics, 2012). Se ha observado que los bebés amamantados tienen un menor riesgo de desarrollar diabetes (Lund Blix et al., 2017), obesidad y enfermedades metabólicas en la vida adulta.

En cuanto a la depresión postparto y a la reducción de peso al lactar, existen controversias relacionadas a los beneficios de la lactancia, ya que ambas condiciones se ven afectadas por variables sociales y conductuales sobre que no se tiene el control (Borra et al., 2015; Feltner et al., 2018). 
Imagen 3. Beneficios de la lactancia en la salud del bebé. Autoría de María Fernanda Tejada Pineda.

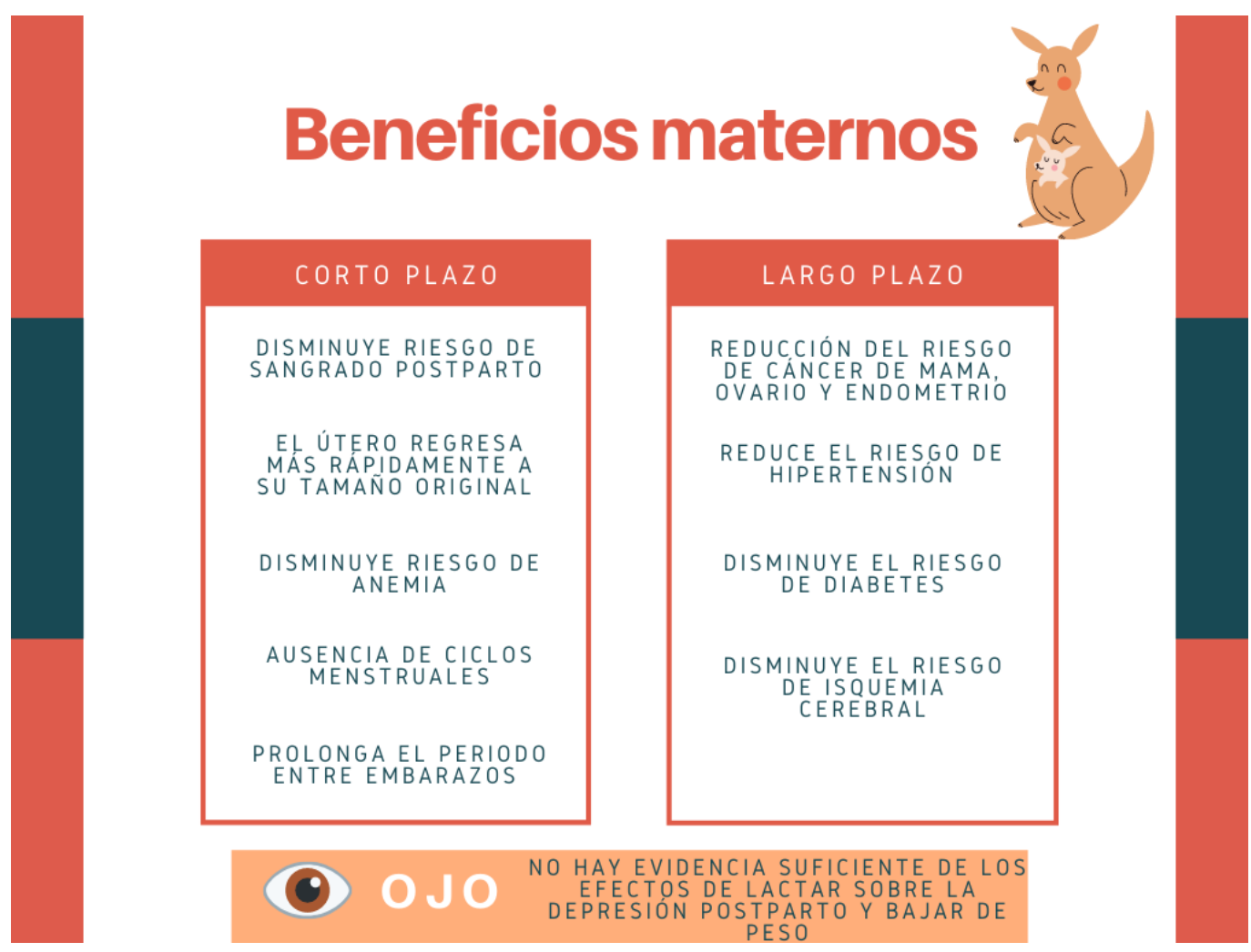

Conclusión

Coexistimos en un mundo, que, hasta el día de hoy, prosigue estigmatizando y/o negando los beneficios de la lactancia en favor de métodos más comercializables. El papel que todos tenemos para pelear con esta tendencia errónea, en el mundo moderno, es multifacético: podemos promover el cambio de políticas públicas para favorecer su imagen, hasta modificar y solicitar condiciones laborales dignas que brinden acceso a lugares privados y seguros para lactar. También, es imprescindible que los prestadores de servicios de salud ofrezcan una educación adecuada a las madres sobre los beneficios de la lactancia, y de igual manera, es necesario que mencionen las ocasiones en las que los riesgos superan a los beneficios y las medidas que se pueden llevar a cabo cuando se imposibilita su realización. De esta forma, se consigue advocar por la decisión informada de la mujer y por su derecho a elegir qué actitud tomar ante la lactancia y, más adelante, cuándo concluirla, lo ideal sería realizarla de manera gradual si no hay una contraindicación absoluta o urgente. La lactancia es, en sí, un tema muy amplio y significativo dentro de nuestro desarrollo, ya que presenta una oportunidad para mejorar la salud materna e infantil, cuestiones prioritarias para la salud pública. 


\section{Referencias}

* Abedi, P., Jahanfar, S., Namvar, F. y Lee, J. (2016). Breastfeeding or nipple stimulation for reducing postpartum haemorrhage in the third stage of labour. The Cochrane Database of Systematic Reviews, 1. https://doi.org/10.1002/14651858.CD010845.pub2

* American Academy of Pediatrics. (2012). Breastfeeding and the Use of Human Milk. Pediatrics, 129(3). https://doi.org/10.1542/peds.2011-3552

- Ballard, O.y Morrow, A. L. (2013). Human milk composition: nutrients and bioactive factors. Pediatric Clinics of North America, 60(1), 49-74. https://doi.org/10.1016/j. pcl.2012.10.002

* Berens, P. (2020). Overview of the postpartum period: Normal physiology and routine maternal care. UpToDate. https://www.uptodate.com/contents/overview-of-thepostpartum-period-normal-physiology-and-routine-maternal-care

* Bonvecchio, A., Théodore, F., González, W., Lozada, A., Alvarado, R. y Blanco, I. (2016). Barreras de la Lactancia Materna en México. En T. González de Cosío Martínez, y S. Hernández Cordero (Eds.), Lactancia Materna en México (77-85 pp.). CONACYT, Intersistemas. https://www.anmm.org.mx/publicaciones/ultimas _ publicaciones/LACTANCIA_MATERNA.pdf

* Borra, C., lacovou, M. y Sevilla, A. (2015). New Evidence on Breastfeeding and Postpartum Depression: The Importance of Understanding Women's Intentions. Maternal and Child Health Journal, 19(4), 897-907. https://doi.org/10.1007/s10995014-1591-z

* Carbajal, R., Veerapen, S., Couderc, S., Jugie, M. y Ville, Y. (2003). Analgesic effect of breast feeding in term neonates: randomised controlled trial. BMJ, 326(7379), 13. https://doi.org/10.1136/bmj.326.7379.13

* Chowdhury, R., Sinha, B., Sankar, M. J., Taneja, S., Bhandari, N., Rollins, N., Bahl, R. y Martines, J. (2015). Breastfeeding and maternal health outcomes: A systematic review and meta-analysis. Acta Paediatrica, International Journal of Paediatrics, 104, 96-113. https://doi.org/10.1111/apa.13102

* Comisión Nacional de los Derechos Humanos (CNDH). (2018). Lactancia Materna: UN DERECHO HUMANO https://www.cndh.org.mx/sites/default/files/doc/Programas/ Ninez_familia/Material/trip-lactancia-materna.pdf

* Diaz, M. (2012). ¿En qué situaciones está contraindicada la lactancia materna? Revista de Pediatría Española, 53, 321-327.

* Feltner, C., Palmieri W.R., Stuebe, A., Grodensky, C., Orr, C. y Viswanathan, A. (2018). Systematic Review of Breastfeeding Programs and Policies, Breastfeeding Uptake, and Maternal Health Outcomes in Developed Countries. Comparative Effectiveness Review, 210. https://effectivehealthcare.ahrq.gov/products/breastfeeding/ research.

* Instituto Nacional de Salud Pública y Fondo de las Naciones Unidas para la Infancia (UnICEF) México. (2016). Encuesta Nacional de Niños, Niñas y Mujeres 2015. https://www.unicef.org/mexico/media/1001/file/UNICEF_ENIM2015.pdf 
* Lund Blix, N. A., Dydensborg Sander, S., Størdal, K., Nybo Andersen, A.-M., Rønningen, K. S., Joner, G., Skrivarhaug, T., Njølstad, P., R., Husby, S. y Stene, L. C. (2017). Infant Feeding and Risk of Type 1 Diabetes in Two Large Scandinavian Birth Cohorts. Diabetes Care, 40(7), 920-927. https://doi.org/10.2337/dc17-0016

* Moore, E.R, Bergman. N., Anderson. G. y Medley, N. (2016). Early skin-to-skin contact for mothers and their healthy newborn infants. Cochrane Database of Systematic Reviews, 11. https://doi.org/10.1002/14651858.CD003519.pub4

* Nguyen, B., Jin, K. y Ding, D. (2017). Breastfeeding and maternal cardiovascular risk factors and outcomes: A systematic review. PIOS ONE, 12(11), e0187923. https:// doi.org/10.1371/journal.pone.0187923

* Organización Mundial de la Salud (oms). (2020). Lactancia materna exclusiva. Organización Mundial de la Salud. https://www.who.int/nutrition/topics/exclusive_ breastfeeding/es/

* Rodríguez García, R. (2015). Aproximación antropológica a la lactancia materna. Antropología Experimental, 15, 407-429. https://doi.org/10.17561/rae.v0i15.2620

- Stiemsma, L. T. y Michels, K. B. (2018). The Role of the Microbiome in the Developmental Origins of Health and Disease. Pediatrics, 141(4). https://doi. org/10.1542/peds.2017-2437

* Talayero, P. (2004). Aspectos históricos de la alimentación al seno materno. En Comité de Lactancia Materna de la Asociación Española de Pediatría(Ed.), Lactancia Materna: guía para profesionales (7-25 pp.). Ergon. https://www.ministeriodesalud. go.cr/gestores_en_salud/lactancia/CNLM_guia_de_lactancia_materna_AEP.pdf

* Victora, C. G., Bahl, R., Barros, A. J. D., França, G. V. A., Horton, S., Krasevec, J., Murch, S., Sankar, M. J., Walker, N. y Rollins, N. C. (2016). Breastfeeding in the 21st century: epidemiology, mechanisms, and lifelong effect. The Lancet, 387(10017), 475-490. https://doi.org/10.1016/S0140-6736(15)01024-7

- Witkowska-Zimny, M. y Kaminska-El-Hassan, E. (2017). Cells of human breast milk. Cellular \& Molecular Biology Letters, 22(1), 11. https://doi.org/10.1186/s11658-0170042-4

\section{Sitios de Interés}

* La lactancia materna (unicef, México)

* 7a Fiesta Mexicana de la Lactancia

\section{Cómo CITAR ESTe ARtículo}

* Velasco Riestra, Paulina, Caicedo Fajardo, Adelaida, Navarro Rodríguez, José Antonio, Tejada Pineda, María Fernanda y Armas Neira, Mónica Jackeline. (2021, julio-agosto). Lactancia materna: rompiendo las barreras. Revista Digital Universitaria (RDU), 22(4). http://doi.org/10.22201/cuaieed.16076079e.2021.22.4.8. 